\title{
Insulator-to-metal crossover near the edge of the superconducting dome in $\mathrm{Nd}_{1-x} \mathrm{Sr}_{x} \mathrm{NiO}_{2}$
}

\author{
Yu-Te Hsu $\odot,{ }^{1, *}$ Bai Yang Wang $\odot,{ }^{2,3}$ Maarten Berben, ${ }^{1}$ Danfeng Li ${ }^{3,4,5}$ Kyuho Lee, ${ }^{2,3}$ Caitlin Duffy $\odot,{ }^{1}$ Thom Ottenbros, ${ }^{1}$ \\ Woo Jin Kim $\odot,{ }^{3,4}$ Motoki Osada $\odot,{ }^{3,6}$ Steffen Wiedmann, ${ }^{1}$ Harold Y. Hwang,${ }^{3,4, \dagger}$ and Nigel E. Hussey ${ }^{1,7, \sharp}$ \\ ${ }^{1}$ High Field Magnet Laboratory (HFML-EMFL) and Institute for Molecules and Materials, \\ Radboud University, Toernooiveld 7, 6525 ED Nijmegen, Netherlands \\ ${ }^{2}$ Department of Physics, Stanford University, Stanford, California 94305, USA \\ ${ }^{3}$ Stanford Institute for Materials and Energy Sciences, SLAC National Accelerator Laboratory, Menlo Park, Stanford, California 94025, USA \\ ${ }^{4}$ Department of Applied Physics, Stanford University, Stanford, California 94305, USA \\ ${ }^{5}$ Department of Physics, City University of Hong Kong, Kowloon, Hong Kong 999077, China \\ ${ }^{6}$ Department of Materials Science and Engineering, Stanford University, Stanford, California 94305, USA \\ ${ }^{7}$ H. H. Wills Physics Laboratory, University of Bristol, Tyndall Avenue, Bristol BS8 1TL, United Kingdom
}

(Received 19 January 2021; accepted 1 October 2021; published 2 November 2021)

\begin{abstract}
We report a systematic magnetotransport study of superconducting infinite-layer nickelate thin films $\mathrm{Nd}_{1-x} \mathrm{Sr}_{x} \mathrm{NiO}_{2}$ with $0.15 \leqslant x \leqslant 0.225$. By suppressing superconductivity with out-of-plane magnetic fields up to $37.5 \mathrm{~T}$, we find that the normal state resistivity of $\mathrm{Nd}_{1-x} \mathrm{Sr}_{x} \mathrm{NiO}_{2}$ is characterized by a crossover from a metallic $T^{2}$ behavior to an insulating $\log (1 / T)$ behavior for all $x$ except $x=0.225$, at which the resistivity is predominantly metallic. The $\log (1 / T)$ behavior is found to be robust against magnetic fields, inconsistent with scenarios involving localization or Kondo scattering, and points to an anomalous insulating state possibly driven by strong correlations. In the metallic state, we find no evidence for non-Fermi-liquid behavior arising from proximity to a putative quantum critical point located inside the superconducting dome.
\end{abstract}

DOI: 10.1103/PhysRevResearch.3.L042015

The realization of superconductivity in infinite-layer nickelates [1] has created a new model system for studying unconventional superconductivity [2-11]. While the defining framework of the high- $T_{\mathrm{c}}$ cuprates, namely a $3 d^{9}$ electronic configuration in square planar geometry, is reproduced in the infinite-layer nickelates, the extent to which the two oxide families can be considered analogous remains an outstanding open question.

The relative strength of the electron correlations, for one, is currently unclear. Undoped cuprates are antiferromagnetic Mott insulators [12]. Undoped bulk $\mathrm{NdNiO}_{2}$, by contrast, does not order down to $1.7 \mathrm{~K}$ [13] while thin film $\mathrm{NdNiO}_{2}$ displays only weakly insulating behavior below approximately $70 \mathrm{~K}$ [1]. The re-entrant insulating behavior found in nickelates at high doping [14-18] further contrasts with the correlated Fermi-liquid ground state found in highly overdoped cuprates [19]. Various theoretical calculations [3-5,20-22, among others] have thus far found that the low-energy electronic structure of infinite-layer nickelates contains contributions from both the $\mathrm{Ni} 3 d_{x^{2}-y^{2}}$ and

\footnotetext{
*yute.hsu@ru.nl

†hyhwang@stanford.edu

${ }^{\ddagger}$ nigel.hussey@ru.nl
}

Published by the American Physical Society under the terms of the Creative Commons Attribution 4.0 International license. Further distribution of this work must maintain attribution to the author(s) and the published article's title, journal citation, and DOI.
$\mathrm{Nd} 5 d$-orbitals-rather than the single-band picture established for the cuprates [12] — though the role or relevance of the $\mathrm{Nd} 5 d$-orbitals remains controversial, particularly in superconducting (SC) samples [23]. X-ray [21], electron energy loss spectroscopy [24], and Hall coefficient $[14,15]$ studies, however, do seem to indicate the presence of multiple bands. Lastly, the peak critical temperature $T_{\mathrm{c}} \approx 10 \mathrm{~K}$ in nickelates is comparatively low, raising the question of whether the two families even share the same pairing mechanism. Evidently, further investigation of the normal state from which nickelate superconductivity emerges is required to ascertain what commonalities, if any, they share with the cuprates.

Typically, the phase diagram of unconventional superconductors harbors a dome of superconductivity bisected by a transition line to some other order, which competes with superconductivity for the dominant ground state [25]. One strategy for determining the evolution of this transition line inside the SC dome and the influence of its termination (at a putative quantum critical point) on the transport properties is to suppress the superconductivity in a high magnetic field and to study the form of the resistivity down to the lowest possible temperatures [26-28]. Here, we present a high-field magnetotransport study on a series of $\mathrm{Nd}_{1-x} \mathrm{Sr}_{x} \mathrm{NiO}_{2}$ (NSNO) thin films with $0.15 \leqslant x \leqslant 0.225$, spanning the entire SC dome. The moderate upper critical field [1] $\mu_{0} H_{\mathrm{c} 2} \lesssim 20 \mathrm{~T}$ in NSNO ensures that the normal state can be accessed in all $\mathrm{SC}$ films down to $T \approx 0.5 \mathrm{~K}$ under continuous magnetic fields up to $37.5 \mathrm{~T}$. At the lowest temperatures, the resistivity undergoes a logarithmic divergence whose magnitude is 

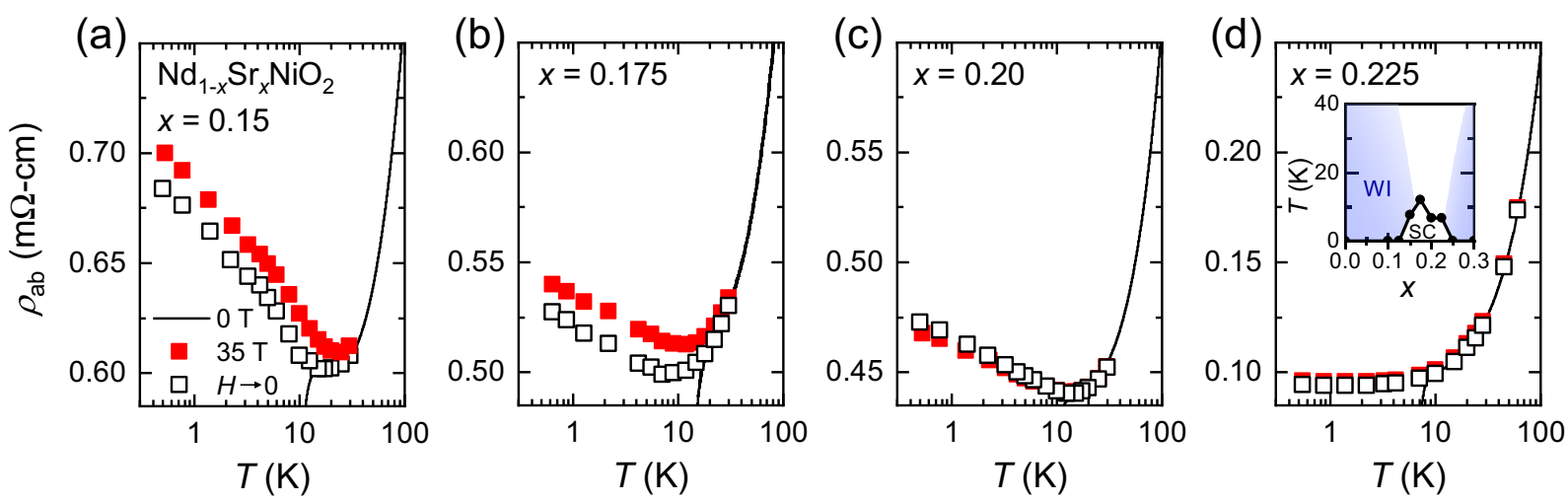

FIG. 1. Semilog plots of the $T$ dependence of the in-plane resistivity $\rho_{a b}$ for (a) $x=0.15$, (b) $x=0.175$, (c) $x=0.20$, and (d) $x=0.225$ measured at zero magnetic field (solid line), at $\mu_{0} H=35 \mathrm{~T}$ (filled squares), and extrapolations from the normal-state magnetoresistance to zero field (open squares). Error bars for $\rho_{a b}(H \rightarrow 0)$ are smaller than the symbols. The inset to panel (d) shows the $T-x$ phase diagram for $\mathrm{Nd}_{1-x} \mathrm{Sr}_{x} \mathrm{NiO}_{2}$, with $T_{\mathrm{c}}$ defined as the midpoint of superconducting transition in the samples shown here. The blue shaded regions represent the weakly insulating (WI) behavior found on both sides of the superconducting dome [14]. Note that the resistivity ranges in each panel are equivalent.

systematically reduced with increasing doping. The resilience of the diverging resistivity to strong magnetic fields excludes localization or Kondo physics as its origin. Its resemblance to the logarithmically divergent resistivity observed in underdoped cuprates [29-31] is noted, however, and suggests that the electronic ground state of infinite-layer nickelates is itself strongly correlated, though possibly not quantum critical. At $x=0.225$, this insulating behavior cedes to a $T^{2}$ metallic resistivity characteristic of a correlated Fermi liquid. Notably, no evidence of non-Fermi-liquid behavior associated with a putative quantum critical point was observed.

$\mathrm{Nd}_{1-x} \mathrm{Sr}_{x} \mathrm{NiO}_{2}$ thin films were grown by pulse laser deposition technique using the high-fluence optimized growth conditions described in Ref. [32]. Electrical resistivity was measured using a four-point method with an alternating current $I=10 \mu \mathrm{A}$ at a frequency between 13 and $30 \mathrm{~Hz}$, with the magnetic fields applied parallel to the crystalline $c$ axis. High-field measurements up to $37.5 \mathrm{~T}$ were performed using a Bitter magnet coupled to a ${ }^{3} \mathrm{He}$ refrigerator at the High Field Magnet Laboratory in Nijmegen, the Netherlands.

Figures 1(a)-1(d) show the normal-state in-plane resistivity $\rho_{a b}(T)$ of four superconducting NSNO thin films with $x=0.15,0.175,0.20$, and 0.225 , respectively. The zero-field normal-state resistivity is extrapolated by fitting the magnetoresistivity isotherms $\rho_{a b}(H)$ shown in Fig. 2(a) using the form of magnetoresistance $\left[\mathrm{MR} \equiv \rho_{a b}(H)-\rho_{a b}(0)\right]$ inferred from the field derivatives (see details in the Supplemental Material [33]). In short, we used a second-order polynomial function to fit the normal state resistivity curve in the highfield range where SC fluctuations are suppressed. At $T>T_{\mathrm{c}}$, the magnitude of the MR is small $(<4 \%$ at $35 \mathrm{~T})$. Below $T_{\mathrm{c}}$, the MR remains small and, crucially, has a negligible $T$ dependence; thus the $T$ dependence of $\rho_{a b}(H \rightarrow 0)$ and normal-state resistivity measured at sufficiently high fields are found to show the same qualitative behavior, independent of the strength of the magnetic field and/or assumed MR functional form (see Figs. S2 and S3 in Ref. [33]). Overall, our conclusions are thus independent of the details of the extrapolation methods.
For $x \neq 0.225, \rho_{a b}(T)$ exhibits metallic behavior down to $T \approx 10 \mathrm{~K}$ before crossing over to insulating behavior with a $\log (1 / T)$ divergence down to $0.5 \mathrm{~K}$. The magnitude of the resistivity divergence diminishes systematically with increasing $x$ and is minimized at $x=0.225$. A similar insulator-to-metal crossover was also observed in the field-induced normal state in various underdoped (UD) cuprates [29-31,34,35]. Indeed, the magnitude of the upturn in NSNO $(\approx 10 \%)$ is comparable to that observed in the electron-doped cuprates $\mathrm{Pr}_{2-x} \mathrm{Ce}_{x} \mathrm{CuO}_{4}$ [34] and $\mathrm{Nd}_{2-x} \mathrm{Ce}_{x} \mathrm{CuO}_{4}$ [35], though it is around one order of magnitude smaller than the corresponding upturns in hole-doped $\mathrm{La}_{2-x} \mathrm{Sr}_{x} \mathrm{CuO}_{4}[29,30]$ and $\mathrm{Bi}_{2} \mathrm{Sr}_{2-x} \mathrm{La}_{x} \mathrm{CuO}_{6+\delta}$ [31]. The origin of the $\log (1 / T)$ resistivity in UD cuprates remains unclear. Nevertheless, it has been noted that low- $T$
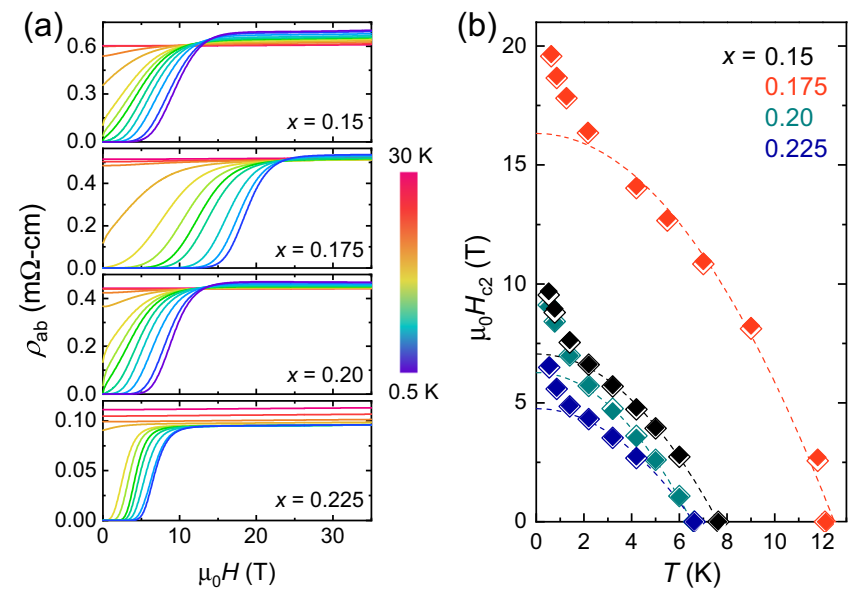

FIG. 2. (a) Resistivity isotherms as a function of magnetic field across the temperature range $0.5 \mathrm{~K} \leqslant T \leqslant 30 \mathrm{~K}$. (b) Upper critical field $H_{\mathrm{c} 2}$ extracted using the $50 \% \rho_{\mathrm{n}}$ criterion (see main text for details). Open and filled symbols correspond to the extractions made using the extrapolated zero-field resistivity and the measured $35 \mathrm{~T}$ resistivity, respectively. Dashed lines are fits to the expression $\mu_{0} H_{\mathrm{c} 2}(T)=\mu_{0} H_{\mathrm{c} 2}(0)\left[1-\left(T / T_{\mathrm{c}}\right)^{2}\right]$ down to $2 \mathrm{~K}$, below which an anomalous upturn in $H_{\mathrm{c} 2}$ is observed. 
upturns in $\rho_{a b}(T)$ are not universal in UD cuprates [36,37], and irradiation studies have indicated that disorder, perhaps in combination with strong correlations, might play the dominant role [38]. The resilience of the resistive upturn in NSNO against magnetic field as well as the positive MR (except for $x$ $=0.20$ ) appears to rule out weak localization, Anderson localization, and Kondo scattering [8] as its origin. The systematic evolution of the upturn in NSNO with doping, coupled with the fact that for $0.15 \leqslant x \leqslant 0.20$, the resistive upturns begin either at or slightly below $T_{c}$, suggests some form of electronic order that competes with the superconductivity for spectral weight, such as the recently reported short-range antiferromagnetic correlations in NSNO [39]. While this conjecture also offers a viable explanation for the evolution of the metallic response (see below), it does not explain the re-entrant weakly insulating state beyond $x=0.225$.

To gain further insight into the low- $T$ electronic ground state of NSNO, we turn to examine the doping dependence of the resistively-determined upper critical field $H_{\mathrm{c} 2}$. Here we use the criterion previously adopted [40] for NSNO, namely the field scale at which $\rho_{a b}(H)$ reaches $50 \%$ of the normalstate resistivity $\rho_{\mathrm{n}}$. This choice is motivated by the reduced impact of SC fluctuations or vortex creep to its functional form [41], despite the fact that the SC order parameter remains finite at this field. Therefore, $H_{c 2}$ shown in Fig. 2 should be considered as the mean-field estimate and a lower bound. As can be seen, $H_{\mathrm{c} 2}$ closely tracks $T_{\mathrm{c}}$ in NSNO, peaking at $\mu_{0} H_{\mathrm{c} 2} \approx 20 \mathrm{~T}$ for $x=0.175$. For each value of $x, H_{\mathrm{c} 2}$ also exhibits an anomalous upturn below $\approx 2 \mathrm{~K}$, consistent with a previous finding [40] for $x=0.225$, possibly indicating an unconventional or multigap SC ground state in the infinitelayer nickelates. We note that, while the choice of a different criterion, e.g., $\rho_{\mathrm{n}, 90 \%}$ or $\rho_{\mathrm{n}, 95 \%}$ changes the magnitudes of $H_{\mathrm{c} 2}$, the qualitative behavior of $H_{\mathrm{c} 2}(T)$ and its evolution with doping remains the same (see Fig. S5 in the Supplemental Material [33]). Curiously, $H_{c 2}$ is found to be the lowest for $x=$ 0.225 . This finding essentially excludes the possibility that the low- $T$ resistivity divergence becomes vanishingly small due to an incomplete suppression of the superconductivity. Rather, the doping-induced insulator-to-metal crossover is characteristic of the field-induced normal-state of the superconducting NSNO investigated here.

The suppression of the resistive upturn as $x \rightarrow 0.225$ provides an opportunity to examine more closely the form of low- $T$ metallic resistivity in NSNO. As shown in Fig. 3, $\rho_{a b}$ for $x=0.20$ can be well fitted to the expression $\rho=\rho_{0}+A T^{2}$ for $300 \mathrm{~K}^{2} \lesssim T^{2} \lesssim 2000 \mathrm{~K}^{2}$ (corresponding to $17 \mathrm{~K} \lesssim T \lesssim$ $45 \mathrm{~K}$ ), the lower bound being set by the onset of the resistive upturn. For $x=0.225, T^{2}$ resistivity is observed only below $7 \mathrm{~K}\left(T^{2} \lesssim 50 \mathrm{~K}\right)$. A pure $T^{2}$ resistivity as $T \rightarrow 0$ is one of the defining signatures of a Fermi-liquid ground state and its extension up to $45 \mathrm{~K}$ in $\mathrm{Nd}_{0.8} \mathrm{Sr}_{0.2} \mathrm{NiO}_{2}$ suggests a predominance of electron-electron over electron-phonon scattering and the presence of strong (unscreened) electron correlations. The same analysis is applied to the other SC concentrations and summarized in Figs. S6 and S7 in the Supplemental Material [33]. While the $T^{2}$ regime is rather narrow for $x<0.20$, due to the growth of the resistivity upturn, we nevertheless find a systematic doping evolution of its onset temperature. To date, there is no doping level at which the low- $T$ resistivity is found (a)

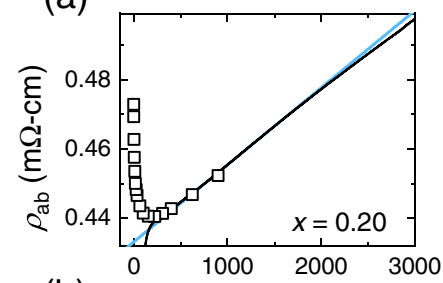

(b)

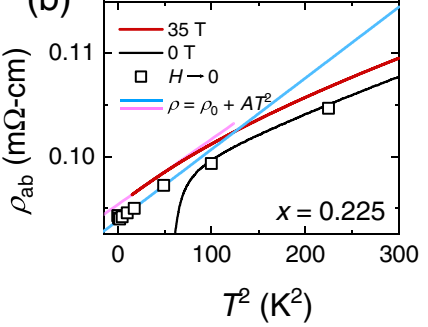

(c)

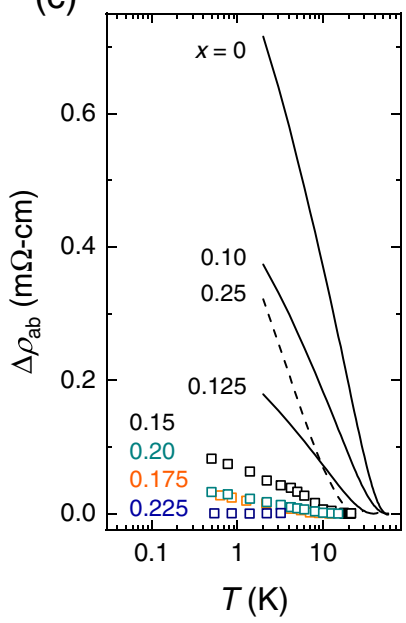

FIG. 3. [(a), (b)] Zero-field resistivity as measured (black lines) and extrapolated (open squares) plotted as a function of $T^{2}$ for (a) $x=$ 0.20 and (b) $x=0.225$. Resistivity measured at $35 \mathrm{~T}$ down to $4.2 \mathrm{~K}$ is also shown for $x=0.225$ (red line). Blue and pink lines are fits made to zero-field and $35 \mathrm{~T}$ resistivity, respectively, using $\rho=\rho_{0}+A T^{2}$. (c) Semilog plot of the resistive upturn $\Delta \rho_{\mathrm{ab}}=\rho_{\mathrm{ab}}-\rho_{\min }$, after the resistivity value at its minimum $\rho_{\min }$ is subtracted from zero-field resistivity. Data of non-SC films are reproduced from Ref. [14].

to have a lower power-law exponent than 2 that might indicate proximity to a quantum critical point. Hence, while there is some evidence from the transport properties of a competing order, it does not, to date, exhibit clear signatures of quantum criticality.

The magnitude of the resistivity upturn $\Delta \rho_{a b}$, defined the difference between $\rho_{a b}(T)$ and the resistivity minimum $\rho_{\min }$, also exhibits a systematic doping evolution across the doping series. As shown in Fig. 3(c), $\Delta \rho_{a b}$ is monotonically suppressed with increasing $x$ until $x=0.225$, at which point, it becomes very small. For $x>0.225, \Delta \rho_{a b}$ again becomes sizable. For lower doped non-SC samples, the resistive upturn is closely correlated with an increase in the Hall coefficient $R_{\mathrm{H}}$ (compare Fig. 1(b) and Fig. 3(a) in Ref. [14]), indicating that the upturn may be due to a drop in carrier density. In SC samples, the small upturns of order 5\% make it difficult to discern any change in $R_{\mathrm{H}}(T)$, particularly if $R_{\mathrm{H}}$ itself has an underlying $T$ dependence in the metallic state. In tandem with the systematic evolution of $\Delta \rho_{a b}(x)$, the drop in carrier density suggests the emergence of a second order parameter that competes with superconductivity for spectral weight, though this is not expected to impose a $\log (1 / T)$ dependence on the resistivity. We note that if the resistive upturn is indeed a signature of charge gapping, the gap, though small in magnitude, must be remarkably robust in the presence of an applied magnetic field. An alternative scenario for the upturn is the development of a secondary scattering mechanism. In this case, the upturn would be an additive contribution to $\rho_{a b}(T)$. In Figs. S7 and S8 in the Supplemental Material [33], we plot the remnant (insulating) contribution to $\rho_{a b}(T)$ after subtracting off a $T^{2}$ component, i.e., $\rho_{\text {rem }}=\rho-\rho_{T^{2}}$ and a $T$-linear component, i.e., $\rho_{\text {rem }}=\rho-\rho_{T}$, respectively. It is clear from Figs. S7 and S8 that the systematic evolution of 
the resistive upturn is not qualitatively changed by our choice of fitting routine.

Curiously, while the metallicity is optimized at $x=0.225$, the strength of the superconductivity in NSNO, as reflected in the absolute magnitude of $H_{\mathrm{c} 2}$, is not. In fact, the $T_{\mathrm{c}}$ and $H_{\mathrm{c} 2}$ are highest at $x=0.175$, showing no clear correlation to the normal state properties in the series of samples investigated here. Evidently, the relation between normal state and superconducting state properties in unconventional superconductors is a complex one, with multiple instabilities vying for spectral weight and frustrating the outcome for the eventual dominant ground state.

In summary, we find a number of salient features of the normal electronic ground state of superconducting nickelates. First, the weakly insulating behavior observed outside the $\mathrm{SC}$ dome is found to persist in the field-induced normal state within the SC regime. Second, at all dopings except $x=0.225$, the low- $T$ resistivity follows closely a $\log (1 / T)$ divergence, behavior reminiscent of that found in various underdoped cuprates [29-31,34,35]. Third, prior to the onset of the upturn, the $T$ dependence of the normal-state resistivity in the metallic regime undergoes a crossover from $T$ linear at high $T$ to $T$ quadratic at lower temperatures. Lastly, at $x=0.225$ where the resistivity divergence is the smallest, the resistivity below $10 \mathrm{~K}$ can be well described by the expression $\rho=\rho_{0}+A T^{2}$, suggestive of the recovery of a correlated Fermi-liquid state. The resilience of the resistivity upturn against magnetic fields appears to preclude localization or Kondo scattering as the origin of the nonmetallic behavior. Rather, it implies the presence of strong unscreened electron correlations, possibly associated with fluctuations of a competing order parameter.

We acknowledge the support of the HFML-RU/NWO, a member of the European Magnetic Field Laboratory (EMFL). This work was supported by the Netherlands Organisation for Scientific Research (NWO) Grant No. 16METL01 “ Strange Metals" and the European Research Council (ERC) under the European Union's Horizon 2020 research and innovation programme (Grant Agreement No. 835279-Catch-22). The work at SLAC/Stanford is supported by the the U.S. Department of Energy, Office of Basic Energy Sciences, Division of Materials Sciences and Engineering, under Contract No. DE-AC02-76SF00515, and the Gordon and Betty Moore Foundation's Emergent Phenomena in Quantum Systems Initiative through Grant No. GBMF9072 (synthesis equipment).
[1] D. Li, K. Lee, B. Y. Wang, M. Osada, S. Crossley, H. R. Lee, Y. Cui, Y. Hikita, and H. Y. Hwang, Superconductivity in an infinite-layer nickelate, Nature (London) 572, 624 (2019).

[2] J. E. Hirsch and F. Marsiglio, Hole superconductivity in infinitelayer nickelates, Phys. C (Amsterdam, Neth.) 566, 1353534 (2019).

[3] A. S. Botana and M. R. Norman, Similarities and Difference Between $\mathrm{LaNiO}_{2}$ and $\mathrm{CaCuO}_{2}$ and Implications for Superconductivity, Phys. Rev. X 10, 011024 (2020).

[4] X. Wu, D. Di Sante, T. Schwemmer, W. Hanke, H. Y. Hwang, S. Raghu, and R. Thomale, Robust $d_{x^{2}-y^{2}}$-wave superconductivity of infinite-layer nickelates, Phys. Rev. B 101, 060504(R) (2020).

[5] E. Been, W.-S. Lee, H. Y. Hwang, Y. Cui, J. Zaanen, T. Devereaux, B. Moritz, and C. Jia, Electronics Structure Trends Across the Rare-Earth Series in Superconducting Infinite-Layer Nickelates, Phys. Rev. X 11, 011050 (2021).

[6] F. Lechermann, Late transition metal oxides with infinitelayer structure: Nickelates versus cuprates, Phys. Rev. B 101, 081110(R) (2020).

[7] M. Jiang, M. Berciu, and G. A. Sawatzky, Critical Nature of the $\mathrm{Ni}$ Spin State in Doped $\mathrm{NdNiO}_{2}$, Phys. Rev. Lett. 124, 207004 (2020).

[8] Y.-H. Zhang and A. Vishwanath, Type-II $t-J$ model in superconducting nickelate $\mathrm{Nd}_{1-x} \mathrm{Sr}_{x} \mathrm{NiO}_{2}$, Phys. Rev. Research 2 , 023112 (2020).

[9] H. Sakakibara, H. Usui, K. Suzuki, T. Kotani, H. Aoki, and K. Kuroki, Model Construction and a Possibility of Cupratelike Pairing in a New $d^{9}$ Nickelate Superconductor (Nd, Sr) $\mathrm{NiO}_{2}$, Phys. Rev. Lett. 125, 077003 (2020).

[10] M.-Y. Choi, W. E. Pickett, and K.-W. Lee, Fluctuationfrustrated flat band instabilities in $\mathrm{NdNiO}_{2}$, Phys. Rev. Research 2, 033445 (2020).
[11] B. Kang, C. Melnick, P. Semon, G. Kotliar, and S. Choi, Infinite-layer nickelates as Ni- $e_{g}$ Hund's metals, arXiv:2007.14610.

[12] P. A. Lee, N. Nagaosa, and X.-G. Wen, Doping a Mott insulator: Physics of high-temperature superconductivity, Rev. Mod. Phys. 78, 17 (2006).

[13] M. A. Hayward and M. J. Resseinsky, Synthesis of the infinite layer $\mathrm{Ni}(\mathrm{I})$ phase $\mathrm{NdNiO}_{2+x}$ by low temperature reduction of $\mathrm{NdNiO}_{3}$ with sodium hydride, Solid State Sci. 5, 839 (2003).

[14] D. Li, B. Y. Wang, K. Lee, S. P. Harvey, M. Osada, B. H. Goodge, L. F. Kourkoutis, and H. Y. Hwang, Superconducting Dome in $\mathrm{Nd}_{1-x} \mathrm{Sr}_{x} \mathrm{NiO}_{2}$ Infinite-Layer Films, Phys. Rev. Lett. 125, 027001 (2020).

[15] S. Zeng, C. S. Tang, X. Yin, C. Li, M. Li, Z. Huang, J. Hu, W. Liu, G. J. Omar, H. Jani, Z. S. Lim, K. Han, D. Wan, P. Yang, S. J. Pennocook, A. T. S. Wee, and A. Ariando, Phase Diagram and Superconducting Dome of Infinite-Layer $\mathrm{Nd}_{1-x} \mathrm{Sr}_{x} \mathrm{NiO}_{2}$ Thin Films, Phys. Rev. Lett. 125, 147003 (2020).

[16] M. Osada, B. Y. Wang, K. Lee, D. Li, and H. Y. Hwang, Phase diagram of infinite layer praseodymium nickelate $\mathrm{Pr}_{1-x} \mathrm{Sr}_{x} \mathrm{NiO}_{2}$ thin films, Phys. Rev. Materials 4, 121801(R) (2020).

[17] M. Osada, B. Y. Wang, B. H. Goodge, S. P. Harvey, K. Lee, D. Li, L. F. Kourkoutis, and H. Y. Hwang, Nickelate superconductivity without rare-earth magnetism: $(\mathrm{La}, \mathrm{Sr}) \mathrm{NiO}_{2}, \mathrm{Adv}$. Mater. 2104083 (2021)

[18] S. W. Zeng, C. J. Li, L. E. Chow, Y. Cao, Z. T. Zhang, C. S. Tang, X. M. Yin, Z. S. Lim, J. X. Hu, P. Yang, and A. Ariando, Superconductivity in infinite-layer lanthanide nickelates, arXiv:2105.13492.

[19] S. Nakamae, K. Behnia, N. Mangkorntong, M. Nohara, H. Takagi, S. J. C. Yates, and N. E. Hussey, Electronic ground state of heavily overdoped nonsuperconducting $\mathrm{La}_{2-x} \mathrm{Sr}_{x} \mathrm{CuO}_{4}$, Phys. Rev. B 68, 100502(R) (2003). 
[20] K.-W. Lee and W. E. Pickett, Infinite-layer $\mathrm{LaNiO}_{2}: \mathrm{Ni}^{1+}$ is not $\mathrm{Cu}^{2+}$, Phys. Rev. B 70, 165109 (2004).

[21] M. Hepting, D. Li, J. Jia, H. Lu, E. Paris, Y. Tseng, X. Feng, M. Osada, E. Been, Y. Hikita, Y.-D. Chuang, Z. Hussain, K. J. Zhou, A. Nag, M. Garcia-Fernandez, M. Rossi, H. Y. Hwang, D. J. Huang, Z. X. Shen, T. Schmitt et al., Electronic structure of the parent compound of superconducting infinite-layer nickelates, Nat. Mater. 19, 381 (2020).

[22] F. Lechermann, Multiorbital Processes Rule the $\mathrm{Nd}_{1-x} \mathrm{Sr}_{x} \mathrm{NiO}_{2}$ Normal State, Phys. Rev. X 10, 041002 (2020).

[23] M. Kitakani, L. Si, O. Janson, R. Arita, Z. Zhong, and K. Held, Nickelate superconductors-a renaissance of the one-band Hubbard model, npj Quant. Mater. 5, 59 (2020).

[24] B. H. Goodge, D. Li, M. Osada, B. Y. Wang, K. Lee, G. A. Sawatzky, H. Y. Hwang, and L. F. Kourkoutis, Doping evolution of the Mott-Hubbard landscape in infinite-layer nickelates, Proc. Natl. Acad. Sci. USA 118, e2007683118 (2021).

[25] T. Shibauchi, A. Carrington, and Y. Matsuda, A quantum critical point lying beneath the superconducting dome in iron pnictides, Annu. Rev. Condens. Matter Phys. 5, 113 (2014).

[26] K. Jin, N. P. Butch, K. Kirshenbaum, J. Paglione, and R. L. Greene, Link between spin fluctuations and electron pairing in copper oxide superconductors, Nature (London) 476, 73 (2011).

[27] J. G. Analytis, H.-H. Kuo, R. D. McDonald, M. Wartenbe, P. M. C. Rourke, N. E. Hussey, and I. R. Fisher, Transport near a quantum critical point in $\mathrm{BaFe}_{2}\left(\mathrm{As}_{1-x} \mathrm{P}_{x}\right)_{2}$, Nat. Phys. 10, 194 (2014).

[28] S. Licciardello, J. Buhot, J. Lu, J. Ayres, S. Kasahara, Y. Matsuda, T. Shibauchi, and N. E. Hussey, Electrical resistivity across a nematic quantum critical point, Nature (London) $\mathbf{5 6 7}$, 213 (2019).

[29] Y. Ando, G. S. Boebinger, A. Passner, T. Kimura, and K. Kishio, Logarithmic Divergence of Both in-Plane and Out-of-Plane Normal-State Resistivities of Superconducting $\mathrm{La}_{2-x} \mathrm{Sr}_{x} \mathrm{CuO}_{4}$ in the Zero-Temperature Limit, Phys. Rev. Lett. 75, 4662 (2020).

[30] G. S. Boebinger, Y. Ando, A. Passner, T. Kimura, M. Okuya, J. Shimoyama, K. Kishio, K. Tamasaku, N. Ichikawa, and S. Uchida, Insulator-to-Metal Crossover in the Normal State of $\mathrm{La}_{2-x} \mathrm{Sr}_{x} \mathrm{CuO}_{4}$ Near Optimum Doping, Phys. Rev. Lett. 77, 5417 (1996).

[31] S. Ono, Y. Ando, T. Murayama, F. F. Balakirev, J. B. Betts, and G. S. Boebinger, Metal-to-Insulator Crossover in the Low-
Temperature Normal State of $\mathrm{Bi}_{2} \mathrm{Sr}_{2-x} \mathrm{La}_{x} \mathrm{CuO}_{6+\delta}$, Phys. Rev. Lett. 85, 638 (2000).

[32] K. Lee, B. H. Goodge, D. Li, M. Osada, B. Y. Wang, Y. Cui, L. F. Kourkoutis, and H. Y. Hwang, Aspects of the synthesis of thin film superconducting infinite-layer nickelates, APL Mater. 8, 041107 (2020).

[33] See Supplemental Material at http://link.aps.org/supplemental/ 10.1103/PhysRevResearch.3.L042015 for additional information on extraction of zero-field resistivity and upper critical fields using different methods, and doping evolution of normalstate resistivity in nonsuperconducting films.

[34] P. Fournier, P. Mohanty, E. Maiser, S. Darzens, T. Venkatesan, C. J. Lobb, G. Czjzek, R. A. Webb, and R. L. Greene, InsulatorMetal Crossover Near Optimal Doping in $\operatorname{Pr}_{2-x} \mathrm{Ce}_{x} \mathrm{CuO}_{4}$ : Anomalous Normal-State Low Temperature Resistivity, Phys. Rev. Lett. 81, 4720 (1998).

[35] S. Y. Li, W. Q. Mo, X. H. Chen, Y. M. Xiong, C. H. Wang, X. G. Luo, and Z. Sun, Low-temperature transport properties of $\mathrm{Nd}_{2-x} \mathrm{Ce}_{x} \mathrm{CuO}_{4+\delta}$ : Metal-insulator crossover in the overdoped regime, Phys. Rev. B 65, 224515 (2002).

[36] F. Rullier-Albenque, H. Alloul, C. Proust, P. Lejay, A. Forget, and D. Colson, Total Suppression of Superconductivity by High Magnetic Fields in $\mathrm{YBa}_{2} \mathrm{Cu}_{3} \mathrm{O}_{6.6}$, Phys. Rev. Lett. 99, 027003 (2007).

[37] C. Proust, B. Vignolle, J. Levallois, S. Adachi, and N. E. Hussey, Fermi liquid behavior of the in-plane resistivity in the pseudogap state of $\mathrm{YBa}_{2} \mathrm{Cu}_{4} \mathrm{O}_{8}$, Proc. Natl. Acad. Sci. USA 113, 13654 (2016).

[38] F. Rullier-Albenque, H. Alloul, F. Balakirev, and C. Proust, Disorder, metal-insulator crossover and phase diagram in high- $T_{c}$ cuprates, EPL 81, 37008 (2008).

[39] H. Lu, M. Rossi, A. Nag, M. Osada, D. F. Li, K. Lee, B. Y. Wang, M. Garcia-Fernandez, S. Agrestini, Z. X. Shen, E. M. Been, B. Moritz, T. P. Devereaux, J. Zaanen, H. Y. Hwang, K.J. Zhou, and W. S. Lee, Magnetic excitations in infinite-layer nickelates, Science 373, 213 (2021).

[40] B. Y. Wang, D. Li, B. H. Goodge, K. Lee, M. Osada, S. P. Harvey, L. F. Kourkoutis, M. R. Beasley, and H. Y. Hwang, Isotropic Pauli-limited superconductivity in the infinite-layer nickelate $\mathrm{Nd}_{0.775} \mathrm{Sr}_{0.225} \mathrm{NiO}_{2}$, Nat. Phys. 17, 473 (2021).

[41] B. Brandow, Characteristic features of the exotic superconductors, Phys. Rep. 296, 1 (1998). 